University of Michigan Law School

University of Michigan Law School Scholarship Repository

1990

\title{
Some Implications of Cognitive Psychology for Risk Regulation
}

\author{
Roger G. Noll \\ James E. Krier \\ University of Michigan Law School, jkrier@umich.edu
}

Available at: https://repository.law.umich.edu/articles/1547

Follow this and additional works at: https://repository.law.umich.edu/articles

Part of the Law and Psychology Commons, and the Public Law and Legal Theory Commons

\section{Recommended Citation}

Krier, James E. "Some Implications of Cognitive Psychology for Risk Regulation." R. G. Noll, co-author. J. Legal Stud. 19 (1990): 747-79.

This Article is brought to you for free and open access by the Faculty Scholarship at University of Michigan Law School Scholarship Repository. It has been accepted for inclusion in Articles by an authorized administrator of University of Michigan Law School Scholarship Repository. For more information, please contact mlaw.repository@umich.edu. 


\title{
SOME IMPLICATIONS OF COGNITIVE PSYCHOLOGY FOR RISK REGULATION
}

\author{
ROGER G. NOLL and JAMES E. KRIER*
}

$\mathrm{B}$ EGINNING with a set of books and articles published in the $1950 \mathrm{~s}^{1}$ cognitive psychologists have developed a new descriptive theory of how people make decisions under conditions of risk and uncertainty. A dominant theme in the theory is that most people do not evaluate risky circumstances in the manner assumed by conventional decision theory-they do not, that is, seek to maximize the expected value of some function when selecting among actions with uncertain outcomes.

The purpose of this article is to consider some implications of the cognitive theory for regulatory policies designed to control risks to life, health, and the environment. ${ }^{2}$ Section I describes the theory and outlines the key differences between it and conventional decision theory. Sections II and III then address, in turn, two central questions about the uses of the theory. First, if people behave in the manner described by the cognitive psychologists, how will this shape the demands that citizens make,

* Noll is Morris M. Doyle Professor of Public Policy, Stanford University; Krier is Earl Warren DeLano Professor of Law, University of Michigan. We thank Bruce Bueno de Mesquita, Amos Tversky, and Barry Weingast for helpful comments on previous versions of this article.

' Identifying the origins of a line of research is always somewhat arbitrary, but we believe that the beginnings of the literature under review here are found in Herbert A. Simon's work on "bounded rationality." See his Models of Man: Social and Rational (1957). A compendium of recent work is Judgment under Uncertainty: Heuristics and Biases (Daniel Kahneman, Paul Slovic, \& Amos Tversky eds. 1982).

2 In his comment on our article, Matthew $\mathbf{L}$. Spitzer observes that the research in cognitive psychology probably has important implications for a host of conventional legal topics. See his Comment on Noll \& Krier's "Some Implications of Cognitive Psychology for Risk Regulation," in this issue. We think Spitzer is right, but now is not the time to accept his invitation to extend our own observations. Interested readers might consider two recent symposia: Legal Implications of Human Error, 59 Univ. S. Cal. L. Rev. 225 (1986); Probability and Inference in the Law of Evidence, 66 Boston Univ. L. Rev. 377 (1986).

[Journal of Legal Studies, vol. XIX (June 1990)]

(C) 1990 by The University of Chicago. All rights reserved. 0047-2530/90/1902-0010\$01.50 
through the political system, for risk regulation, and how (if at all) might these demands differ from those that would be expected if citizens behaved, instead, in the manner assumed by conventional decision theorists? Second, if citizens make demands as predicted by the cognitive theory, how (if at all) might their behavior affect the regulatory responses that political actors supply?

These questions are not entirely new to the risk assessment literature, but our approach to them is novel in that we seek to integrate the cognitive psychologists' theory of decision making under uncertainty with the political economists' theory of policy-making and implementation in a representative democracy. Thus far, the literature on cognitive psychology and risk regulation has mostly focused on two concerns: (1) redefining the normative criteria used to evaluate risks and policy responses to them, ${ }^{3}$ and (2) examining how citizen opinion on risk policy issues is affected (and perhaps manipulated) by the manner in which the issues arise and are discussed in the political arena. ${ }^{4}$ The primary aim of the first kind of work has been to enrich the methods of risk analysis; the primary aim of the second has been to augment our understanding of how political rhetoric influences the process of issue information. ${ }^{5}$

Our concerns are different. We seek to identify how, in a democratic society, public attitudes about risk might influence the kinds of risk regulation programs that will be enacted, given that political officials, in their quest for electoral security, seek to satisfy the preferences of constituents. This task presents us with something of a dilemma. Like much of life generally, politics itself is a risky business. If the cognitive psychologists are correct in describing how people perceive and make decisions about hazards to their well-being, then their model may also apply just as fully to political actors-actors who will be mindful, after all, that their policy decisions will have an (uncertain) effect on their desire to secure reelection or otherwise advance their careers. Alternatively, political competition may cause people who use decision theory to win more elections than people who behave as the cognitive theory describes. A comprehensive treatment of the questions we pose would thus have to consider how the cognitive theory alters our understanding of the behavior of

${ }^{3}$ See, for example, Paul Slovic, Baruch Fischhoff, \& Sarah Lichtenstein, Regulation of Risk: A Psychological Perspective, in Regulatory Policy and the Social Sciences 241 (Roger G. Noll ed. 1985).

${ }^{4}$ See, for example, Baruch Fischhoff, Ola Svenson, \& Paul Slovic, Active Responses to Environmental Hazards, in 2 Handbook of Environmental Psychology 1089 (D. Stokols \& I. Altman eds. 1987).

5 See esp. George A. Quattrone \& Amos Tversky, Contrasting Rational and Psychological Analyses of Political Choice, 82 Am. Pol. Sci. Rev. 719 (1988). 
both citizens and elected political officials, compared to the conventional view.

For now, though, this task is too ambitious. We choose to proceed on the assumption that while cognitive theory accurately describes how citizens make decisions about risks to life and health, traditional decision theory can be aptly applied to the political actor's problem of calculating the best response to citizen demands for action. That said, let us state here a point expanded on in Section IV's concluding remarks. Cognitive theory offers a number of apparent insights into human behavior and regulatory policy that differ from or go beyond those generated by the standard decision-theoretic model of the economics and politics of regulation. The insights are derived from experiments and surveys, many of which are controversial because of their design. We do not attempt to assess the strength and validity of the insights here, our chief concerns being description and elaboration. We are struck, though, by the large number of insights from the cognitive theory that are consistent with conventional analysis. In other words, although the cognitive theory is novel for its hypotheses about how people perceive risk and act in response to it, the hypotheses themselves have few novel implications for regulatory policy.

\section{Cognitive Decision Theory}

In this section, our purpose is simply to describe some empirical findings by cognitive psychologists that are inconsistent with the general assumptions of conventional decision theory. We shall not deal in detail with the attempts of others to incorporate the cognitive theory into standard decision analysis. ${ }^{6}$ Moreover, we are not concerned with observations by the cognitive psychologists that have to do with how people evaluate events as a function of the details of the risks they pose. (Examples include evaluations based on distinctions between types of risk, such as voluntary vs. involuntary, identifiable vs. statistical, and concentrated vs. diffuse. Factors like these, which ultimately hinge on the risk attributes people care most about, can easily-if tautologically-be included in the traditional decision-theoretic model, though commonly they are not.)

Our focus is on three elements of cognitive theory: prospect theory, heuristics and biases, and cognitive pathologies. Prospect theory refers to a set of hypotheses about the mathematical form of the functions describing how individuals evaluate risky outcomes; cognitive theorists offer the

${ }^{6}$ Mark J. Machina has written an informative survey of this work. See his Choice under Uncertainty: Problems Solved and Unsolved, 1 J. Econ. Persp. 121 (1987). 
hypotheses as alternatives to the mathematical forms used in decision theory. Heuristics and biases refer to cognitive shortcuts people use to solve complex problems. The shortcuts cause "mistakes" in the sense that they lead to decisions inferior to the decisions that would have been reached had the shortcuts not been used. Use of the shortcuts is not necessarily irrational, however, because it saves information-processing and decision-analysis costs. If the errors induced by heuristics and biases are sufficiently small on average, and if the information and decision costs are sufficiently large, then the use of shortcuts can yield net benefits to decision makers. Cognitive pathologies are systematic, repeated decision methods (not random errors) that are simply irrational and mistaken. They consist of presuppositions and calculation methods that do not conserve on information and decision costs and that can be expected to impose losses on decision makers.

In comparing these elements of cognitive theory to conventional decision theory, we do not attempt to include literally every aspect of them that has been identified or talked about in the literature. Instead, we try to capture the thrust of cognitive theory by revealing its essentials and dwelling on the aspects that have the most important implications for riskmanagement policy.

The baseline for our comparative treatment is conventional expected utility theory, which has the following core assumptions. ${ }^{7}$

First, people evaluate any action in terms of the level of welfare it produces. Welfare is usually conceptualized as the unmeasurable value of a utility function, whose arguments are quantitative measures of economic variables such as wealth, income, or consumption. The utility function is typically assumed to obey the law of diminishing marginal utility, which implies risk aversion on the part of the decision maker.

Second, if the outcomes of an action are uncertain, people evaluate the action according to its expected value. To perform such an evaluation, the decision maker is said first to envision outcomes as a set of mutually exclusive, collectively inclusive events and then to assign to each event an estimate of its relative likelihood that obeys the laws of probability. Then the decision maker calculates the expected value, or probabilityweighted sum, of all of the possible outcomes of a given action.

Third, estimates of the probabilities and values of the outcomes of each action are efficiently calculated, making the best use of available information. For example, new information about the likelihood outcomes leads to a recalculation of the probabilities according to Bayes's Rule.

\footnotetext{
${ }^{7}$ For a comparison of the key assumptions of expected utility and much of cognitive theory, see $i d$.
} 
A formal representation of these three assumptions facilitates a comparison of expected utility with the cognitive theory. Suppose that some action can lead to one of three possible wealth outcomes, $X, Y$, and $Z$, having probabilities $p, g$, and $r$, respectively, where $p+q+r=1$ and where $p, q$, and $r$ are all nonnegative. Let $v(\cdot)$ be the evaluation (utility) function of wealth. For purposes of decision analysis, the evaluation, $V$, of the action in question is given by

$$
V=p v(X)+q v(Y)+r v(Z) .
$$

Moreover, if some level of wealth $W$ satisfies

$$
V=v(W),
$$

then, because of the law of diminishing marginal utility,

$$
W<p X+q Y+r Z \text {. }
$$

Suppose there exists some event that, if it occurs, means the final wealth state will be either $X$ or $Y$. If the event occurs, the probabilities of the wealth states must be recalculated, and their new values, $p^{\prime}, q^{\prime}$, and $r^{\prime}$, are

$$
p^{\prime}=p /(p+q), \quad q^{\prime}=q /(p+q), \quad r^{\prime}=0 .
$$

The revised value of the action is then

$$
V^{\prime}=[p v(X)+q v(Y)] /(p+q) \text {. }
$$

Finally, an important property of decision theory is that the evaluation process exhibits rational expectations. Essentially, this means that intervening events that either have been anticipated or are irrelevant to the consequences of a subsequent action will not alter the decision maker's choice of subsequent actions. A formal example that proves useful for comparative purposes is as follows. Suppose a decision maker can select either of two actions, $A$ and $B$, with evaluations $V_{A}$ and $V_{B}$, where $V_{A}>$ $V_{B}$. Suppose also that the decision maker faces two problems that are identical, save that in the first the valuations of actions $A$ and $B$ are as described above, while in the second the values of each action will only be available if some other event (with a probability $p$ ) occurs. In the case of the first problem, the decision maker selects action $A$ because $V_{A}$ is larger than $V_{B}$. In the case of the second problem, the expected values are as follows:

$$
V_{A}^{*}=p V_{A}+(1-p) 0,
$$

and

$$
V_{B}^{*}=p V_{B}+(1-p) 0 .
$$


Obviously, if $V_{A}$ is larger than $V_{B}$, then $V^{*}{ }_{A}$ is also larger than $V^{*}{ }_{B}$; hence, the choice of actions should be independent of an intervening probabilistic event. The decision maker would make the same choice whether the decision between $A$ and $B$ is made before or after this event. The reason is that the event provides no information about the relative values of the two actions.

The attack of cognitive psychologists on the traditional decisiontheoretic model sketched above has three components. First, empirical evidence indicates that people do not use valuation functions over outcomes of the form $v(X)$ described above. Second, other empirical evidence indicates that decisions about actions are not based on probabilityweighted sums of even descriptively more accurate forms of the valuation function. Third, people do not respond to new information in the ways described by Bayes's Rule and rational expectations.

\section{A. Outcome Valuation Functions}

From their experimental evidence, cognitive psychologists hypothesize that valuation functions do not contain only the outcomes of actions. Thus, they propose a function exhibiting a "reference effect," where the valuation of an outcome depends on the sign and magnitude of the change in the status quo that the outcome would produce. ${ }^{8}$

The reference effect has two components, loss aversion and the reflection effect. ${ }^{9}$ Loss aversion refers to the fact that people ascribe additional negative value to an outcome if it represents a negative change from the status quo. Thus, the valuation function contains the status quo, the direction of change, and the magnitude of change and has the property that, for baseline $Y$ and change $X$,

$$
v(Y, 0)>v(Y+X,-X) .
$$

The reflection effect works in a direction opposite to that of loss aversion but is never fully offsetting. It postulates that, for increases in income, $v$ obeys diminishing marginal utility, but, for decreases in income, $v$ exhibits increasing marginal utility. Thus, descriptively, people are risk averse as to gains but risk taking as to losses. Consider a lottery with probability $p$ of being at a level of wealth $(Y+X)$ and with probability ( 1 $-p$ ) of being at wealth $Y$. According to the cognitive theory, there must be some level of wealth $W$, between $Y$ and $Y+X$, such that

$$
v(W)<(1-p) v(Y, 0)+p v(Y, X),
$$

${ }^{8}$ Daniel Kahneman \& Amos Tversky, Prospect Theory: An Analysis of Decision under Risk, 47 Econometrica 263, 286-88 (1979).

${ }^{9}$ Id. at 268, 287; Quattrone \& Tversky, supra note 5, at 720-21. 
and

$$
v(W)>(1-p) v(Y+X,-X)+p v(Y+X, 0) .
$$

Using traditional decision theory, the right side of each of these expressions collapses to $(1-p) v(Y)+p v(Y+X)$, so that both inequalities cannot be true simultaneously.

Another observation of cognitive psychologists is that people are not particularly effective in estimating outcome values. The process by which people go from the description of an outcome to its evaluation is affected by a phenomenon called coding, or the use of various heuristics as evaluation shortcuts. ${ }^{10}$ (The notion is that people like to avoid careful consideration of alternatives and so seek ways to simplify the process.) Although coding has many aspects, two are especially important in considering behavioral responses to risk. One of these is the representativeness heuristic, which refers to a tendency to reason by analogy to previous circumstances. ${ }^{11}$ Thus, if on superficial observation an outcome $Y$ appears to be similar to an outcome $X$, people tend to set $v(Y)=v(X)$. This is not particularly problematic when outcomes can be aptly expressed in a single dimension; the representativeness heuristic is troublesome, however, when outcomes have multiple attributes because equating the outcomes on the basis of only some attributes can lead to evaluation errors. Moreover, because errors vary as a function of the attributes actually inspected, the errors themselves are random and unpredictable in the absence of a systematic search method (but predictable when a systematic search procedure is used).

The other important aspect of coding is the tendency to ignore small differences through the use of rounding ${ }^{12}$ and ratio-difference principles. ${ }^{13}$ Rounding refers to the practice of regarding some complex quantity as the nearest simple one (for example, forty-nine and fifty-one are both rounded to fifty). Ratio difference refers to thinking of changes in magnitude in percentage terms and treating a given percent of a big number as equivalent to the same percent of a small number.

An important element of the cognitive psychological theory of outcome valuation is called the framing effect. ${ }^{14}$ The manner in which people value outcomes depends on how an outcome is characterized or presented

10 Kahneman \& Tversky, supra note 8, at 274.

11 David Grether, Bayes' Rule as a Descriptive Model: The Representativeness Heuristic, 95 Q. J. Econ. 537 (1980).

12 Kahneman \& Tversky, supra note 8, at 275.

13 Quattrone \& Tversky, supra note 5, at 727-28.

${ }^{14}$ Id. at 727-30. 
("framed"): Is it a gain or a loss? Is it the result of a sequence of small changes or one summary large change? Is the baseline against which change is measured defined narrowly, making the change large in percentage terms, or defined expansively, making the change small in percentage terms? In economic analysis, the appropriate concept for calculating the costs of an action is the value of the benefits (opportunities) forgone by taking that action rather than the next best alternative. When dealing with certain costs and benefits, of course, the convention concerning which action is the "status quo" (and hence the source of opportunity costs) and which is the "proposed action" (and hence the source of benefits) is unimportant. But when benefits and costs are uncertain, cognitive decision theory predicts that the convention of presentation, or framing, does matter.

\section{B. The Calculation of Action Values}

Cognitive decision theory also claims that evaluating actions on the basis of the expected values of outcomes is incorrect, even if the valuation functions are altered to take into account the factors described above. The claim has two major components. First, people are said to systematically misestimate probabilities. Second, the weights they place on outcomes are not equal to their probability estimates.

The first assertion is based on observations of a variety of circumstances in which people do not make the best use of available information in estimating probabilities. One persistent source of error in this regard arises from the availability heuristic, whereby people are influenced in estimating the probability of an event by whether an event similar to that in question comes readily to mind. ${ }^{15} \mathrm{~A}$ similar event might come readily to mind because it occurred recently; for example, plane crashes are thought to be more probable by people who have just heard of one. Or a similar event might come readily to mind even though it occurred long ago because of selective recollection of, say, pleasant memories (nostalgia). In this way, people believe old movies are likely to be better than new ones because all of the old movies they remember were good ones. Another source of error is anchoring, referring to a tendency to resist altering (to the extent required by Bayes's Rule) a probability estimate, once formed, when pertinent new information comes to light. ${ }^{16}$ Finally, a third source of error arises from overconfidence: people tend to underestimate the degree to which their own knowledge and judgments are imperfect. ${ }^{17}$ An impor-

15 Slovic, Fischhoff, \& Lichtenstein, supra note 3, at 245-47.

${ }^{16}$ Grether, supra note 11.

${ }^{17}$ Slovic, Fischhoff, \& Lichtenstein, supra note 3, at 247-48. 
tant example of overconfidence is neglecting possible events and outcomes that might influence the value of an action and then (because all probabilities sum to one) overestimating the probabilities associated with the outcomes that do come to mind.

To summarize, people will tend to overestimate the probability of an outcome if an example of the event has recently been called to their attention or otherwise comes readily to mind, if recent information indicates that the outcome has become less likely, or if a large number of other outcomes could occur but each is quite unlikely by itself and infrequently comes to mind. Probabilities will tend to be underestimated if the opposite of each of these instances is the case.

Once people form probability estimates, cognitive decision theory holds that they will not use them directly to calculate the expected value of actions. Instead, they will behave as though they were using another set of weights. Moreover, the precise weighting method that is used will depend on the characteristics of the decision to be made. These ideas are drawn together under the name prospect theory. ${ }^{18}$

Prospect theory begins by addressing how the weights used in evaluating actions are calculated. If $p$ represents a person's belief about the actual value of a probability (perhaps calculated incorrectly, as described above), then $P(p)$ is the weighting function that translates $p$ into the weights used in calculating the value, $V$, of an action. In general, $P(p)$ has the following properties. ${ }^{19}(a) P(p)>p$ if $p$ is very small, but for other $p$ 's, $P(p)<p$. (b) Nonconcatenation: for probabilities $p$ and $q, P(p q)>$ $P(q) P(p)$ for small values of $p$, but $P(p q)<P(q) P(p)$ otherwise. (c) Subcertainty: if $p, q>0$ and $p+q=1, P(p)+P(q)<1$. (d) Certainty effect: $P(0)=0$ and $P(1)=1$. In properties $a$ and $b$, "small" is not precisely defined but is generally thought to apply to probabilities below approximately 10 percent.

The implications of these assumptions are as follows. First, people behave as if they think that low-probability events are more likely than their own beliefs about the probabilities would suggest; for all other larger probabilities less than unity, however, they behave as if the events were less likely than they believe. Moreover, if an event of given probability is the consequence of one source of uncertainty, people behave as if it is more likely to occur than if it is the consequence of several sources of uncertainty but has the same concatenated probability. Conceptually, nonconcatenation suggests that people apply an added discount to concatenated probabilities because "there are so many ways to lose" com-

18 Kahneman \& Tversky, supra note 8.

19 Id. at 275-76, 280-84. 
pared to the "one way to lose" from a single event with the same objective probability. Finally, subcertainty is a mechanism whereby risk aversion can enter action evaluations other than through the outcome valuation functions. Not only do valuation functions satisfy "loss aversion" and, for gains, diminishing marginal utility, but, in addition, weighted sums of outcomes are further depressed by a tendency to have their weights sum to less than their actual probabilities.

Two other implications of the prospect function, $\boldsymbol{P}$, are potentially important but have not been examined in the literature on prospect theory. One implication is that the degree of subcertainty (that is, the difference between unity and the sum of the weights) is not completely independent of the values taken by the probabilities. The general shape of the function $P(p)$ is assumed to be linear, except that, to satisfy all of the conditions placed on it, the function must have some nonlinear regions. For example, $\boldsymbol{P}(p)$ must be relatively flat for low values of $p$, but more steep for high values of $p$, in order to satisfy all of the conditions placed on it (as described in our preceding discussion). This implies that, for lotteries involving one very unlikely event plus some events with higher probabilities, the sum of the weights on outcomes declines as the probability of the unlikely event increases. Consequently, people behave as if they assign a value nearer to the actual expected value of a lottery if it contains a highly unlikely event. Thus, the "extra" risk aversion implied by the form of the $P(p)$ function is greater when all of the events are regarded as sufficiently likely that $P(p)<p$ for all outcomes.

A second unnoticed aspect of the presumed shape of the $P(p)$ function is that it works in a direction opposite to that of the overconfidence hypothesis. Specifically, if the sum of outcome weights is less than unity, the effect behaviorally is as if the decision maker had assigned a positive probability to another possible outcome that is not incorporated into the decision problem. The valuation of this "phantom" outcome is not necessarily zero, but to explore this issue requires examining how prospect theory hypothesizes that weights and outcomes are combined to evaluate action.

Contrary to conventional decision theory, prospect theory does not assume that actions are evaluated simply by summing the weighted values of outcomes. Only if at least one possible outcome preserves the status quo, or if at least two outcomes entail changes from the status quo of opposite sign, will decision makers evaluate actions analogously to calculating expected value. In this case, if an action can produce three possible changes in the status quo, $X, Y$, and $Z$, with probabilities $p, q$, and $r$, respectively ( $p+q+r=1$ ), and if $I$ is the status quo, then the evalua- 
tion of the action is given by

$$
\begin{aligned}
V= & P(p) v(I, X)+P(q) v(I, Y)+P(r) v(I, Z) \\
& +[1-P(p)-P(q)-P(r)] v(I, 0) .
\end{aligned}
$$

If an action leads to outcomes that all change the status quo in the same direction, then decision makers are assumed to proceed as follows. First, they determine which outcome involves the smallest departure from the status quo. Second, they assume that the outcome involving the smallest departure will occur with certainty. Third, they calculate the value of the otner outcomes as uncertain departures from the status quo. The effect of this procedure is to overstate the relative contribution to the value of the action that is caused by the outcome producing the smallest departure. Formally, for an action involving an initial position $I$, three possible changes in the status quo $(X, Y$, and $Z$, all of which are either strictly positive or strictly negative, and among which $X$ has the smallest absolute value), and probabilities $p, q$, and $r$ (which sum to one), prospect theory proposes that actions will be evaluated according the following calculations: ${ }^{20}$

$$
\begin{aligned}
V= & v(I, X)+P(q)[v(I, Y)-v(I, X)] \\
& +P(r)[v(I, Z)-v(I, X)]+[1-P(q)-P(r)] v(I, 0) .
\end{aligned}
$$

An interesting feature of both of the foregoing formulas is that they accord weight to the status quo even though, by assumption, the status quo has zero probability of continuing. Technically, this appears to create a logical inconsistency in the theory since the certainty effect requires $P(0)=0$. However, this exception to the certainty effect reflects another essential component of the theory, the reference effect, according to which the value of all uncertain actions depends on the value the decision maker places on the status quo.

The implication of equation (2) is that the outcome involving the smallest departure from the status quo is given more weight than $P(p)$. The reason is that, with subcertainty, $P(p)<1-P(q)-P(r)$; however, the latter weight is implicitly given to $v(I, X)$ in the first expression. If $q$ and $r$ are "not small," this implies that the weight accorded to $v(I, X)$ is larger than the subjective probability. If $p$ is "small," then $v(I, X)$ is doubly overweighted-partly because $P(p)$ exceeds $p$, and partly because [1 $P(q)-P(r)]$ exceeds $P(p)$. Finally, if $q$ or $r$ is "small," the implicit weight on the smallest change may be larger or smaller than its subjective proba-

${ }^{20}$ Id. at 276. 
bility. The reason is that the overweighting of the low-probability event is offset to an unknown extent by the substitution for $P(p)$ in the evaluation function.

The bearing of the preceding observations is that, if all outcomes of an action involve some loss, two types of outcomes are accorded excessive weight in the evaluation of actions. First, for outcomes in which the loss is small, there occurs what might be called the "optimism effect." Given a range of possible catastrophes, the best among a bad lot will tend to have too much influence on the evaluation of an action. Second, as emphasized above, large catastrophic outcomes with nontrivial probabilities will be undervalued, whereas large catastrophic outcomes with low probabilities will be overvalued. Note that, in the second case, the valuation function over losses already has a shape that encourages risk-taking behavior, so the action evaluation process serves to enhance the likelihood that people will be willing to take relatively high chances of very large losses.

The evaluation function when events do not produce results of the same sign has a similar but slightly different implication. If one alternative, say $X$, is zero (that is, it maintains the status quo), then the first formula accords the status quo a weight of $1-P(q)-P(r)$, which is not only larger than $P(p)$ but is also larger than $p$ unless both $q$ and $r$ are "small." Two kinds of plausible action-outcome relations fit this case. In one, an action may have "large" probabilities of both gain and loss, in which case both outcomes are relatively undervalued compared to the status quo. This has the effect of offsetting risk aversion (including loss aversion); however, it has no necessary implication concerning the willingness to undertake such an action. In the other action-outcome relation, the socalled Faustian bargain in which a high probability of small gain is paired with a low probability of catastrophe, the latter is overweighted relative to the former (this might be called the "pessimism effect"). Note, then, an interesting implication of prospect theory: people may be excessively prone to avoid actions that can have both good and bad consequences, but when faced with the certainty of some loss, they may be excessively prone to take actions involving a reasonably large chance of a catastrophic outcome.

An important prediction from prospect theory is an aversion to "probabilistic insurance," meaning an action that reduces but does not eliminate the probability of a loss. ${ }^{21}$ In general, the theory predicts a dislike for insurance because of the risk-taking shape of the valuation function over losses. Moreover, because insurance converts a risk from a situation in which one outcome is good (a loss of zero) to a situation in which all outcomes entail a loss (the smallest of which is loss of the insurance

21 Id . at 269-71. 
premium), the presence of loss aversion and, in the former case, an overweighting of the status quo further militate against insurance. ${ }^{22}$ In addition, if probabilistic insurance reduces a "large" risk to a "small" one, the weighting of the bad outcome will not be reduced in proportion to its reduced probability. For all of these reasons, people are likely to prefer either no insurance at all or insurance that always protects totally against the loss. In particular, insurance that reduces the probability of loss from a reasonably large one to a very small one will be relatively undervalued. Of course, here, "insurance" can mean any costly action that reduces the chance of a bad event.

Prospect theory also predicts actions inconsistent with the idea of rational expectations. This can be demonstrated by a simple example. Suppose a person has an initial position $I$ and is indifferent between the certainty of $X$ or a chance $p$ of $Y$, implying that $v(I, X)=P(p) v(I, Y)+[1$ - $P(p)] v(I, 0)$. (In this example, we will henceforth suppress argument $I$ for ease of notation.) Now consider a case with two probabilistic events. In the first event, a person receives either nothing with probability $q$, or, with probability $(1-q)$, the chance to experience the second event, which entails receiving either $X$ or a chance $p$ of $Y$. The choice between $X$ and $p$ of $Y$ must be made before the outcome of the first event is observed. To select between these options requires a comparison between the following values:

$$
V_{x}=P(1-q) v(X)+[1-P(1-q)] v(0),
$$

and

$$
V_{y}=P[(1-q) p] v(Y)+\{1-P[(1-q) p]\} v(0) .
$$

If $V_{x}$ and $V_{y}$ are equal, as rational expectations theory requires, then, with rearranging, the following must hold:

$$
v(X) \stackrel{?}{=} \frac{P[(1-q) p] v(Y)+\{P(1-q)-P[(1-q) p]\} v(0)}{P(1-q)} .
$$

But from the first problem,

$$
v(X)=P(p) v(Y)+[1-P(p)] v(0) .
$$

Hence, both of these relationships can hold only if

$$
P[(1-q) p]=P(1-q) P(p) .
$$

But this violates the nonconcatenation property of prospect theory; hence, both equalities cannot hold.

22 Howard Kunreuther et al., Disaster Insurance Protection: Public Policy Lessons (1978). 
This significance of the preceding example is this. In some cases, people will prefer a guaranteed outcome to a lottery. However, if they must decide whether to play the lottery or take the certain outcome, they may express the opposite preference and commit to a lottery in advance of knowing whether the commitment will ever amount to anything. But then, if events transpire such that their choice has a bearing and their commitment is binding, they will regret not having taken the certain outcome. The implication is that people will be prone not to protect in advance against risks that may not emerge, even though, if the risks do emerge, people will regret their decision, notwithstanding that the objective relative merits of the safe and risky options have not been affected.

\section{ImPlications Regarding the Demand for Risk Regulation}

To consider how the cognitive theory of decisions under risk translates into demands for political action, we first must specify the theory of political processes that maps citizen preferences into incentives to act on the part of government officials. We will begin by exploring the implications of the "positive responsiveness" of majority-rule electoral processes, then amend the treatment to take account of "mobilization bias." Our interest in both cases will focus on two inquiries: first, how citizen demands, given the cognitive view, will depart from those predicted by traditional decision theory; and second, what kinds of decisions are especially susceptible to the possibility of preference reversal with the unfolding of time. The second inquiry has to do with circumstances in which citizens change their minds about the appropriateness of some policy, even though the objective conditions surrounding the policy choice have not changed. These circumstances are especially important to government officials because they create the possibility for intertemporal inconsistencies in citizen demands. A political leader caught in such circumstances has to make a trade-off between disappointing citizens today and disappointing them tomorrow, when their preferences have changed. How politicians might deal with these problems is the subject of Section III.

\section{A. Pure Majority-Rule Democracy: Positive Responsiveness}

One characteristic of majority-rule decision processes is that the likelihood of a policy being adopted does not decline as the number of citizens who favor it increases, a property called positive responsiveness. ${ }^{23}$ In analyzing the policy implications of cognitive decision theory, we first

${ }^{23}$ William H. Riker, Liberalism against Populism, ch. 3 (1982). 
focus on this property. We will not deal extensively with the other major property of majority rule-its fundmental instability. In general, the outcome of a system of pairwise, majority-rule votes depends on the particular sequence in which the alternatives are considered. ${ }^{24}$ Without restrictions on sequence, majority rule can usually lead to essentially any policy outcome, and any policy that wins at one moment can be defeated at the next vote. ${ }^{25}$

The importance of the instability of voting mechanisms for our purposes is that it provides risk-averse voters and political leaders with an incentive to design political systems in which policies are difficult to change once adopted. Such an idea, called structure-induced equilibrium, ${ }^{26}$ has been offered as one rationale for the complexity of the American federal system (multiple independent levels of government, checks and balances in the federal structure) and the rules of procedure governing elections and legislative processes. The key point is that political institutions designed to combat the inherent instability of majority-rule democracy also attenuate the responsiveness of policy to changes in citizen preferences. Hence, if citizens exhibit intertemporal inconsistencies in preferences regarding risk regulation, modifications in policy are unlikely fully to reflect these changes in preferences.

The first type of risk-management problem that arises involves an ongoing, valued activity that also carries with it the possibility of causing serious harm. Here, cognitive theory indicates that people will tend to value the activity more highly than its objective expected value as long as the probability of the damaging event is not too low. They will take risks contraindicated by conventional decision theory. But if the probability of the damaging event becomes sufficiently small, the opposite result is obtained. People will seek to terminate an activity that traditional decision theory indicates should be continued. The overall effect, from the perspective of decision theory, is to take too many risks of high probability and too few of low probability.

This effect influences expenditures to ameliorate risks, that is, to bear the costs of some measure that would reduce the probability or magnitude of damage. In general, as the probability of a damaging event increases, so too will the amount that citizens are willing to spend to ameliorate it. But, unlike traditional decision theory, the willingness to pay for amelioration rises less rapidly than the rate of increase in the probability of the

\footnotetext{
24 Id., ch. 5.

25 Kenneth Arrow, Social Choice and Individual Values (1951).

${ }^{26}$ Kenneth A. Shepsle \& Barry R. Weingast, Structure-induced Equilibrium and Legislative Choice, 37 Pub. Choice 503 (1978).
} 
damaging event. From the decision theory perspective, citizens are willing to spend relatively too much on low-probability events as compared to ones of higher probability.

If the foregoing preferences are based on accurate estimates of probabilities and outcome values, then they are unlikely to present intertemporal instability problems for government officials. In essence, those sorts of problems arise because true preferences persistently and systematically diverge from the evaluations arising from the application of traditional decision theory. In this case, the occurrence of a damaging event at a frequency consistent with its objective probability will not cause a citizen's evaluation of policies for dealing with it to change over time.

Circumstances change if citizen preferences are affected by the cognitive pathologies discussed earlier, such as availability, anchoring, representativeness, and overconfidence. Now, the occurrence (or lack of occurrence) of a damaging event can alter the estimates of probabilities, the valuation of outcomes, or both. Moreover, the extent to which preferences are likely to shift over time is predictable.

Consider, for example, the effect of availability, which predicts that people will systematically overestimate the probability of an event if similar events come readily to mind but will systematically underestimate them otherwise. The implication is that, immediately following a widely publicized disaster, citizens will place unusually great demands on their government to take action against recurrence, but as attention subsides, so too will the demand for action.

An interesting implication of prospect theory is that intertemporal instability is less of a problem in the case of events with small probabilities. The reason is that the weighting function $P(p)$ is less sensitive to changes in $p$ when $p$ is small; if citizens alter their estimates of $p$ because of recent experience, relatively small changes in the evaluation of policies for dealing with the risk will result. Thus, policies regarding low-probability events are likely to be more resistant to changes in perceptions about the underlying risk than policies dealing with more likely events.

A second set of risk regulation issues is related to the violation of rational expectations in cognitive decision theory. Imagine the decision to develop a viable new technology that, after the research and development $(\mathrm{R} \& \mathrm{D})$ phase, can be implemented in either a risky form (high economic returns but a chance of catastrophe) or a safe form (lower returns but few, if any, risks). One possibility is to postpone the development decision until after the $R \& D$ phase. The other possibility is to make a decision between the safe or risky form during the $R \& D$ phase. The first strategy allows the decision about safety to be taken after the technology is known to be commercially interesting, whereas the second strategy requires 
commitment to a decision about safety before commercial feasibility is established. As discussed in Section I, interposing the uncertainty of commercial feasibility may well reverse the choice between safe and risky options, such that, after the $\mathrm{R} \& \mathrm{D}$ phase is complete, citizens will regret the commitment made before $\mathrm{R} \& \mathrm{D}$ began, even though no new information about the nature of the risk and the cost of ameliorating it has become available.

The underlying inconsistency here can result in regretting either choice. The safe alternative can be regretted, ex post, because resolving the uncertainty about commercial feasibility increases the expected value of the risky option, as calculated by prospect theory. Recall that, at the low end, an increase in objective probability causes a less-than-proportional increase in the outcome weight for evaluating options, whereas, for higher probabilities, this relationship is more nearly proportional. For this rather common case-a high probability of no accident, a small probability of damage-risk policy would be expected to exhibit greater caution before commercial uncertainties are resolved than after. This provides a reason why greater caution is generally exercised in licensing new industrial ventures than in regulating established ones.

But preference reversal can work the other way too. Before the fact, a new technology may be regarded as a lottery with both good and bad outcomes; after the technology has been put in place, all of the outcomes may be perceived as favorable. The reason is that, initially, the $R$ \& $D$ cost may be lost if the technology turns out to be commercially infeasible; however, if commercial feasibility is demonstrated, then the possibilities can become (1) a risky plant that has no accidents; (2) a risky plant that has an accident but that, nevertheless, proves to be, on balance, a social benefit; or (3) a safe plant with a net benefit intermediate between the two. The second case demonstrates more risk aversion than the former, according to prospect theory, such that, in stage 1 , the risky strategy may be preferred. In stage 2 , if commercial feasibility is proved, that decision may be regretted. This tendency would be amplified by the availability heuristic if the damaging event occurred after a plant was put into operation.

A third issue affected by the cognitive theory is the trade-off between making a decision now and postponing the decision until more information is acquired. The motivation to wait is to obtain new information to resolve uncertainties about probabilities, the magnitude of damages, or the costs of protection against risks. Cognitive theorists have not fully explored this issue, but a natural interpretation is that people will calculate the value of information in much the same way as in decision theory, except that the calculations will be based on updated outcome weights 
(not updated probabilities) using the evaluation formulas of prospect theory. The key observations here are that the outcome weights do not change in proportion to the changes in probabilities and that the nature of the lottery can change if information is received that switches the applicable evaluation formula.

Outcome weights increase relatively slowly for low-probability events and have discontinuities at probabilities of zero and one-with the following implications. First, information that serves to refine estimates of low probabilities will be accorded less value than in decision theory, given the less-than-proportional relationship between outcome weights and actual probabilities at the low end. Second, information conclusively showing that an event is either certain or of zero probability has an accentuated value, relative to decision theory, owing to the discontinuities in the weighting function at extreme probabilities. Third, for issues that do not involve low probabilities, both good and bad outcomes will be treated in a roughly symmetrical fashion; however, because probability weights are less than probabilities across the board and sum to less than one, the general tendency is to undervalue information. Refining these probabilities (assuming that certainty is not obtained) will still change values by less than would be the case if all calculations were based on actual probabilities.

The conclusion to be reached is that, in general, the value of information is likely to be lower if people behave as cognitive theory claims rather than according to traditional decision theory. Complete certainty is an unlikely product of any search for more information; so, in most realistic cases, information will be accorded too low a value. This tendency is reinforced by some other behavioral characteristics identified by cognitive theory. For example, anchoring implies that probabilities are updated less than they ought to be as new information appears, and overconfdence suggests that people underestimate the extent to which there are uncertainties to be resolved. The implication of these considerations is that information will be given a still lower value than that suggested by the formal calculations of prospect theory.

Even if information is acquired, the consequences are not likely to be what one might conventionally expect. Essentially, information theory asks people to examine what decision they will make after an uncertainty has been resolved. Rational expectations theory can be invoked to argue that people can accurately assess how they will behave in such circumstances, but prospect theory says people violate rational expectations. Even if information is acquired, people might renege on their agreements about how they would respond to it. The acquisition of information presents basically the same problem as the decision about a safe or risky 


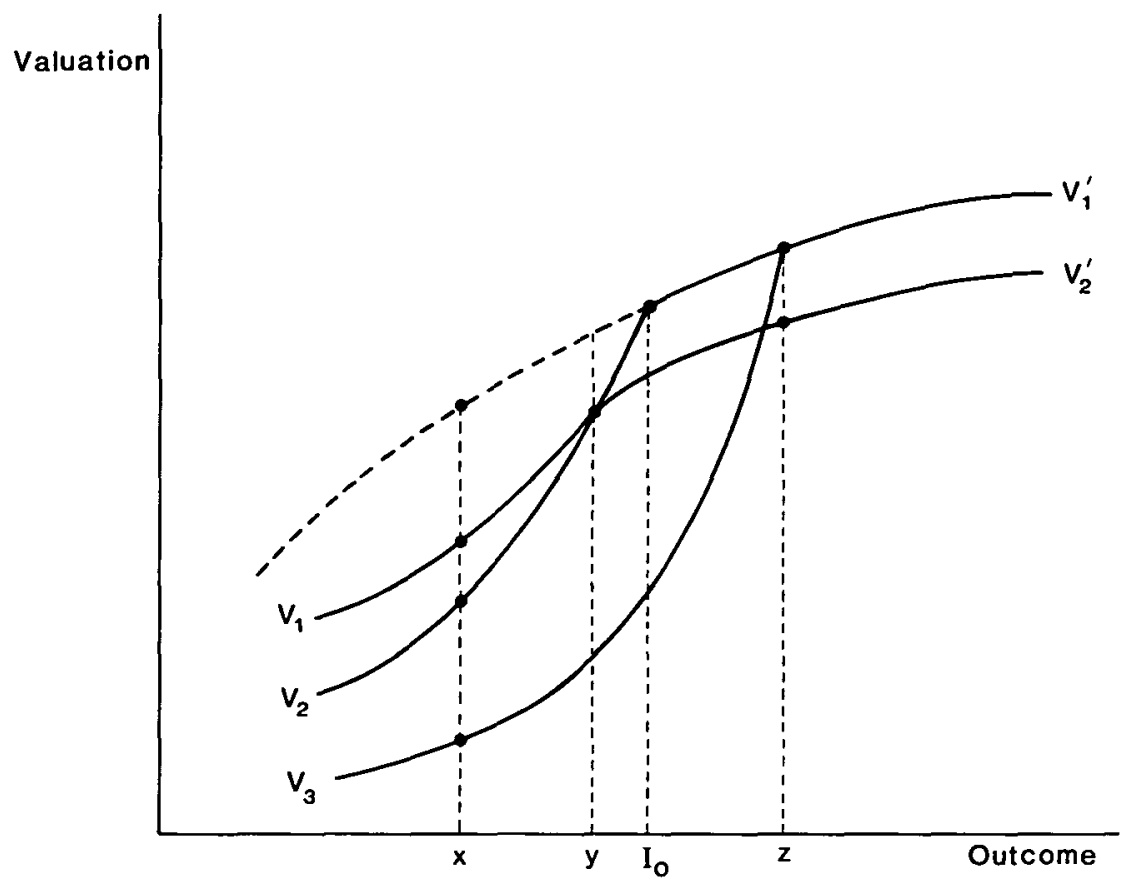

Figure 1

technology prior to knowledge about its commercial feasibility. Resolving the uncertainty about the results of the information search can reverse people's preferences.

A final aspect of the cognitive theory that affects preferences regarding risk-management problems involves the path dependence of preferences. Path dependence arises from the hypothesis that individual valuations of changes in outcomes (or utility functions) are asymmetrical around the status quo, exhibiting risk aversion in gains and risk acceptance in losses. Thus, as the outcomes moves away from the status quo in either direction, the ratio of the change in value (utility) to the change in outcome is largest for the smallest changes. The implication is that both the shape and the position of the valuation function depend on the status quo-that is, individual valuations of a particular outcome depend on the status quo ante from which that outcome was obtained. The nature of path dependence in the cognitive theory, and its implications for behavior, can be clarified by a diagram.

Figure 1 depicts the valuation function over outcomes under prospect theory. Note that "loss aversion" over the entire range of feasible 
changes from the status quo requires that the actual valuation function over losses must always lie below the projection of the valuation function over gains into the range of losses. In the figure, this projection is the dotted line between initial state $I_{0}$ and outcomes producing worse outcomes, such as $X$ and $Y$. That is, the actual valuation function $V_{1} V_{1}^{\prime}$ lies below the dotted projection for all feasible outcomes below $I_{0}$. Moreover, it has a shape that bows in a direction opposite that of the projection.

Note also the effect of an actual bad outcome, which replaces $I_{0}$ and $Y$. The latter outcome becomes the new status quo, and the valuation function is redefined from this baseline, as depicted in line $V_{2} V_{2}^{\prime}$. The implication is that, after $Y$ has transpired, all feasible outcomes except $Y$ now have a lower value than they had when $I_{0}$ was the initial point. Likewise, if a good outcome transpires-such as point $Z$ - the portion of the valuation function below $Z$ is altered to reflect loss aversion below that point, so that the new valuation function becomes $V_{3} V_{1}^{\prime}$. Thus, the bad outcome $X$ produces a lower valuation if it is reached from a higher initial point. More generally, the valuation of a bad outcome keeps getting worse as the value of the status quo increases, and the value of a good outcome is lower for lower values of the status quo.

Figure 1 reveals the path dependence of outcome values: precisely the same risk can produce different evaluations, depending on the status quo and on the sequence of events that produced the status quo. Suppose, in the figure, that the comparison is between a lottery of $X$ or $Z$ and the certainty of $I_{0}$. Clearly, the valuation of the relative merits of these choices depends on whether the status quo is $I_{0}$ or $Y$ (after a fall from $I_{0}$ ). In the latter case, the valuation of the lottery has fallen relative to the certainty of $I_{0}$ because the shape of the valuation function causes larger drops in the valuation of $X$ and $Z$ than in the valuation of $I_{0}$. Alternatively, if the choice is between the same lottery and the current status quo $\left(I_{0}\right.$ or $Y$ ), the lottery has been made more attractive owing to the fall from $I_{0}$ to $Y$. After this fall, the lottery gives the person more to gain and less to lose.

In most instances, the outcomes of policy decisions regarding environmental risk take time to materialize. Hence, a citizen will evaluate a policy with reference to the status quo prevailing at the time of the evaluation. If, for example, incomes are increasing (or citizens, for other reasons, regard themselves as better off as time progresses), an environmental risk that appears worth taking today will lead to regret in the future. Moreover, if environmental risk policy consists, in general, of a series of alternating gains and setbacks, people will regard themselves as becoming steadily worse off in some cases where, by objective measures of outcome, they are actually becoming better off. Note that, in the figure, the outcome $Z$ along $V_{2} V_{2}^{\prime}$ is objectively superior, but subjectively worse, 
than the outcome $I_{0}$ along $V_{1} V_{1}^{\prime}$. Hence, the sequence $I_{0}: Y: Z$ makes people feel worse off than they would had they remained at $I_{0}$. Obviously, this means that, if the cognitive theory is correct, environmental risk policy must either avoid significant setbacks altogether or be very good indeed, overall, if people are to perceive policy progress. The result is somewhat analogous to the distaste for probabilistic insurance: policies that serve to reduce (but not to eliminate) the losses due to occasional damaging events can simultaneously increase the average value of realized outcomes as measured objectively but leave people with the perception that, on balance, they are worse off. The reason is the extra welfare cost associated with any loss-a cost that, according to the cognitive theory, has a permanent depressing effect on a person's future ability to derive welfare from good outcomes.

\section{B. Political Economy and the Cognitive Theory}

Real representative democracies are more complex than the simple majority-rule system hypothesized in the preceding section. Decisiontheoretic models of the political process emphasize two key elements of political participation: rational ignorance and mobilization bias. ${ }^{27}$ Rational ignorance refers to the lack of incentives on the part of citizens to be fully informed about the policy positions a candidate advocates in an election campaign. The incentive problem is caused, first, by the mismatch between the complexity of policy and the simplicity of the signal a voter can send (yes, no, or abstain) and, second, by the powerlessness of a single vote. To acquire and digest information requires time and effort, yet after exhaustive analysis a voter can send only a very weak signal, one that is not sensitive to the intensity of the voter's preferences, and one that is highly unlikely to influence the outcome of an election. In consequence, there is little incentive to compare candidates and their policies carefully. Voters are likely to pay relatively little attention to campaigns and will usually base their decisions primarily on information freely available in an entertaining form (TV sound bites) - and even then will pay attention only to messages concerning a small number of issues that they regard as especially important (such "salient" issues as the state of the economy and of international relations, plus a few more specific matters of particular interest to a given voter). Voting behavior should be especially likely to exhibit the kinds of cognitive shortcuts described in Section I.

Mobilization bias refers to the fact that some preferences tend to be more effectively represented in political processes than others. One way

27 Anthony Downs, An Economic Theory of Democracy (1957). 
to overcome rational ignorance is to rely on someone else to perform issue analyses, then to vote as instructed. Similarly, one way to overcome voter powerlessness is to act in concert with other like-minded individuals. ${ }^{28}$ Collectively, a group of voters might have a substantial chance of swinging an election to one candidate or another. Moreover, through campaign contributions, a group can help preferred candidates provide the free entertaining information that might lead others to vote as the group desires. But forming groups, devising strategies, and coordinating efforts also require time, effort, and money. Hence, small, homogeneous groups with high per capita stakes in an issue are more likely to organize successfully than are large, heterogeneous groups with small per capita stakes. $^{29}$

We can now consider how the cognitive theory provides some insights into the bearing of rational ignorance and mobilization bias on risk policies.

Framing, discussed in Section 1, suggests that, in a world of rational ignorance, the way political actors describe issues will alter a voter's evaluation of policy options. For example, if an issue is put as one involving alternative ways to obtain gains, voters will exhibit risk aversion; whereas, if the same issue is framed in terms of various ways to experience losses, voters will exhibit loss aversion with risk-taking behavior. ${ }^{30}$ Hence, the way an issue is stated can influence outcomes. Consider the case of a proposal to make seat belts mandatory, with a mandatory fine if one is caught unbelted. Outcomes could be expressed variously as follows:

(a) a large benefit from driving without a belt, not being caught, and having no accident;

(b) a somewhat smaller net benefit from driving belted and having no accident;

(c) a smaller net benefit still from driving unbelted, getting caught and fined, and having no accident;

(d) a small negative net driving benefit from wearing a belt and having an accident;

(e) a more negative net benefit from driving unbelted and having an accident; and

$(f)$ a slightly more negative net benefit from driving unbelted, having an accident, and being fined to boot (adding insult to injury).

\footnotetext{
28 Mancur Olson, The Logic of Collective Action (1965).

29 Roger G. Noll \& Bruce M. Owen, The Political Economy of Deregulation, ch. 3 (1983).

${ }^{30}$ Quattrone \& Tversky, supra note 5.
} 
Facing these possibilities, a voter is likely to exhibit risk aversion because some possibilities are expressed positively.

Alternatively, one could put the seat-belt issue in terms that start with a status quo in which driving safely, unbelted, and unfined is the baseline, so that all other outcomes are negative. This would induce risk-taking behavior and a propensity to search for accident-reduction policies that reduce risks to zero (to avoid probabilistic insurance). In short, adeptness at characterizing issues can go a long way in determining the policy preferences expressed by the electorate. ${ }^{31}$

Issue salience is also related to issue presentation. According to the representativeness heuristic, one can induce preferences concerning policies about one risk by presenting it as being like another risk. Then, voters will evaluate the two risks as though they were the same. Therefore, if one environmental risk becomes salient (because, say, a damaging event has recently occurred), political actors may piggyback other environmental risks on the first by presenting them as essentially identical to the salient one.

The availability effect accentuates the importance of salience. A damaging event, if timed appropriately and if widely publicized, induces people to behave as if the likelihood of such events had increased. If the effect makes the issue politically salient-makes it one of the few issues that can animate political participation-then it can serve, for a time, to help overcome mobilization bias. With regard to environmental risk policy, risk producers will normally be the better-organized, more effective participants in the political process. But, a highly publicized environmental catastrophe can cause some citizens to overestimate the degree of risk they face and thus to be spurred to mobilize for political action. Moreover, other political actors may enhance the success of their own mobilization efforts by clever use of representativeness in the presentation of other issues. Mobilization bias may be temporarily overcome-temporarily because, if the probability of some damaging event is truly low, then events of that sort are likely gradually to subside from public consciousness and lose their salience. ${ }^{32}$

${ }^{31}$ Spitzer, supra note 2, at text around note 6, asks us to indicate how voters choose the status quo. We do not know, and we are in good company. As Camerer points out in his comment on our article, "frame choices are hard to predict" (Colin F. Camerer, Comments on Noll and Krier, "Some Implications of Cognitive Psychology for Risk Regulation," in this issue, at text around note 4): For some speculations on frame choices from a sociologist's point of view, see Carol A. Heimer, Social Structure, Psychology, and the Estimation of Risk, 14 Ann. Rev. Soc. 491 (1988).

${ }^{32}$ For examples of these observations, drawn from the history of air pollution control efforts in Los Angeles in the years after World War II, see James E. Krier \& Edmund Ursin, Pollution and Policy 263-77 (1977) (discussing the effect of salient "crises" on pollution policy and how they wane over time). 
The mechanics of how availability and mobilization bias work on a class of risk policy issues are complex, but a few prototypical examples provide some insight into the general process. Consider three types of low-probability risk circumstances. One is a low-probability catastrophic event that, if it occurs, will harm a large number of people simultaneously, such as the Bhopal or Chernobyl disasters. The second is an event in which the probability of a disaster at a facility is again low, but where there are a large number of facilities. Assume here that the number of people harmed per disaster would be much lower than in the first case, but large enough to make for a newsworthy event. Examples are airline or bus accidents, where between a few and a few hundred people are killed or injured. Events of the second type occur more frequently than those of the first type; assume that the lower damage per event is exactly offset by the greater exposure, so that the average annual number of deaths would be the same in the two cases. The third type of event is one presenting a risk to only a single person or a very small group. Events of this sort again have low probability, but a very large number of exposures causes frequent occurrences-so frequent that, with such small losses per event, they are not newsworthy.

Availability suggests a different politics for each of the three types of events, even if they have the same expected losses. Regarding the first, most of the time the issue is not before the public, so policy questions are not salient. The dominant form of politics here will be mobilization bias, with well-represented groups who have atypically high stakes in the issue serving as the major drivers of policy. But immediately after an event actually occurs, it will become very salient because of the large amount of damage, and this will cause more broad-based political demand for substantial ameliorative action.

In the second case, the higher frequency of events, all of which pass the availability threshold, will tend to keep the issue perpetually salient to people other than those already well organized into groups. Policy here is less likely to be prone to alternating periods of hectic corrective fire drills and quiescence. Organized interests will be influential in the quiescent periods, but frequent periods of salience, anticipated by political actors, will tend to work against complete capture even in quiet times.

In the third case, risks are never salient, so policy will either be nonexistent or dominanted entirely by interests with atypical stakes.

We can think of examples for each of these extremes. Great earthquakes are very low probability events (even in California) that threaten large disasters. Yet even in areas that are relatively earthquake prone, enforcement of building standards (especially their retroactive application to old structures) is lax. Airline crashes are relatively frequent, newswor- 
thy events, and airline safety regulation is especially stringent, making it by far the safest mode of transportation in terms of expected annual fatalities per unit of exposure. By contrast, safety standards and their enforcement are quite lax in the case of automobiles and motorcycles, resulting in higher accident rates than those of other modes of transportation in which greater numbers of people are at risk in any given accident.

In the view of some observers, comparisons like these reveal a citizen preference for greater safety when large numbers of people are simultaneously at risk. This may be true, but, even in the absence of that particular preference, the cognitive theory would predict the same pattern of political demands-except in the case of truly calamitous outcomes. In this instance, the theory is rather ambiguous. On the one hand, availability will suppress the demand for policy in this arena most of the time. On the other hand, the tendency to overweight low-probability events in calculating action values will have a compensating effect. How these two effects balance out is, of course, not predicted by the theory. What the theory does predict is intertemporal inconsistency in the demand for policy, with the occurrence of an event causing a temporary surge of demand.

\section{Summary of Political Demands for Risk Policy}

The preceding discussion leads to several generalizations about the kinds of demands citizens collectively will make on government with regard to risk policies.

1. The intensity of demand for policies to ameliorate risks will tend to be higher for low-probability risks and lower for high-probability risks than is predicted by conventional decision theory.

2. The intensity of demand for policies to ameliorate risks will depend on whether the risk is perceived as a lottery among different levels of improvement, among different levels of losses, or among a mixture of gains and losses.

Imagine that a previously unsuspected potential source of risk is suddenly discovered-say through the invention of a beneficial, but risky, new technology. Suppose that, without controls, the technology offers a lottery between small and large gains but, with controls, brings the certainty of a moderate gain. Here, demand will tend to reflect risk-averse behavior. Suppose now, in the alternative, that new information appears about previously unsuspected dangers from an established valuable technology. The choice of what to do requires selection among a range of costly controls that would reduce, but not eliminate, the amount or likelihood of damage, or both. In this case, demand will tend to exhibit risk- 
taking behavior. Consider, finally, a new technology that may bring benefits but may also bring net reductions in welfare. Here, loss aversion will tend to overcome risk taking in losses. If, however, the possible gains and losses are both very large, citizen preferences may exhibit risk-taking behavior in the selection between partial and total control.

3. The intensity of demand for risk policies will diverge from the predictions of conventional decision theory depending on the frequency and magnitude of consequential events, holding constant the expected and the actual long-term average losses.

The demand for policy will be affected, first, by the fact that the weights assigned to outcomes increase less than proportionately to outcome probabilities, so that events with lower frequency but with outcome values of compensatingly greater magnitude are not evaluated equally. In addition, availability will mitigate against demand for risk-avoidance policies at two extremes: very bad outcomes of very low probability and very frequent events with very small damages.

4. Policy preferences will exhibit intertemporal instability, even if underlying information about outcomes and probabilities does not change.

Intertemporal instability has two sources. First, decision evaluations depend on whether there are intervening inconsequential events between the decision and the outcome and, incidentally, on the status quo (which determines which outcomes are net gains and which are net losses). Second, intertemporal instability arises from framing effects in political discourse because people's perceptions of risks and policies to ameliorate them are determined by the language used to describe the issues.

5. The effect of new information on policy preferences depends on how familiar citizens are with the risk-with overreaction to new information in the case of unfamiliarity and underreaction in the case of considerable familiarity. Essentially, if people update expectations as the cognitive theory assumes, one consequence is that expectations are subject to the availability effect when an event is unfamiliar and to overconfidence or anchoring when it is familiar.

6. Rational ignorance (nonsalience) and mobilization bias are offset to some degree for low-probability events by the form of the weighting function and for all harmful events by the availability effect (temporarily after an event transpires). In other periods, the absence of an occurrence enhances the effects of rational ignorance and mobilization bias.

\section{Implications Regarding the Supply of Risk Regulation}

The premise of the following discussion is that, if the cognitive decision theory presents an accurate picture of human behavior, then elected polit- 
ical officials will take into account its effects on the political preferences of their constituents when making policy. We assume that officials seek to secure reelection and that they adopt policies with that end in mind. Political actors may, of course, have their own personal objectives to pursue, but first they have to succeed with the electorate. So we will focus on reelection-maximizing strategies, given the lessons of cognitive theory about electorate behavior.

The first challenge facing elected political officials is to institutionalize systematic differences in the stringency of risk regulation among types of risks so as to reflect the different evaluations of the risks by citizens. (Some risks will be more intensively controlled than others, as measured, say, in terms of expenditures per unit of reduction in expected harm.) One way to accomplish this end would be through a statutory specification of differential objectives. This approach can, however, create problems in several respects. First, changes in preferences, knowledge of risks, or technological developments can quickly make the original policy targets obsolete, requiring time-consuming and sometimes unpredictable policy reauthorization. ${ }^{33}$ Second, the intertemporal inconsistencies predicted by prospect theory guarantee that a specific statement of a policy objective will be regarded as suboptimal either now or in the future (and perhaps both). Third, if different risks affect different members of the electorate, explicit differences in the commitment to protecting them may provide effective ammunition for future political challengers who can use the issue to appeal to disfavored groups. ${ }^{34}$

A more effective means for producing differential policy outcomes is to specify ambitious targets in all programs but vary resource allocations and the procedural requirements for adopting regulations among various risk-reduction programs. ${ }^{35}$ For example, one way to make airline safety standards more rigorous than auto safety standards is simply to give the agency regulating airlines a much larger budget and much more statutory authority, relative to the problem it is asked to solve, than that given to auto regulators. In the case of toxic chemicals, ${ }^{36}$ the Food and Drug Administration (FDA) has, in the Delaney amendment, a very strong and

33 Mathew D. McCubbins, Roger G. Noll, \& Barry R. Weingast, Structure and Process, Politics and Policy: Administrative Arrangements and the Political Control of Agencies, 75 Va. L. Rev. 431 (1989).

34 Riker, supra note 23 , ch. 8.

35 Roger G. Noll, The Political Foundations of Regulatory Policy, 139 Zeitschrift fur die gesamte Staatewissenchaft 377 (1983).

36 Mathew D. McCubbins, Roger G. Noll, \& Barry R. Weingast, Administrative Procedures as Instruments of Political Control, 3 J. L., Econ., \& Org. 243, 268-69 (1987). 
easily invoked tool for removing man-made carcinogens from foods, whereas the Toxic Substances Control Act (administered by the Environmental Protection Agency [EPA]) provides only a weak mechanism for keeping off the market new toxic chemicals used in industrial processes. The government bears the burden of proving that a substance is carcinogenic in both cases, but the standard of proof is lower for the FDA than for the EPA. Of course, these differences do not necessarily have their basis in cognitive decision theory; they merely demonstrate how resource allocations and statutory language could lead to policies of quite different stringency among different categories of risk.

Procedural tools are also a potentially useful way to deal with intertemporal instability in preferences. For example, if the demand for policy reflects availability-because it arises from some catastrophic event unlikely to recur soon-strong policy rhetoric, combined with a protracted process and an understaffed agency, assures that the actual standards for ameliorating the risk will be adopted by the implementing agency only long after the event in question has occurred. Detailed regulatory procedures administered by a resource-poor agency thus allow politicians to "lash themselves to the mast" while waiting out the temporary siren calls for immediate overreaction; they allow an agency to "strike when the iron is cold," after the issue has lost its political salience.

The strategies of lashing to the mast and striking when the iron is cold are politically effective but not necessarily normatively correct. The normative problem arises from the fact that, as salience wanes in the course of the agency's developing its regulatory strategy, participation in the policy process will come to be dominated by organized interests, usually the industries subject to regulation. Hence, a publicly interested policy enacted in the midst of public outcry and relatively free of mobilization bias will end up being implemented through a regulatory process hidden from view and subject to special interest favoritism. This may appear to be politically advantageous to elected officials in that it allows them to serve public demands today and special interest desires tomorrow, but even here a problem arises. If an event similar to that in question is likely to recur sometime during the career of politicians responsible for the regulatory policy, then they risk being blamed for unsuccessful regulation. Given this risk, the best political strategy might be to enact rather rigorous and explicitly specified policy targets but set up a process of implementation that provides distributive benefits to participants in the process. An example would be a cumbersome process for setting industry standards, combined with differentially more rigorous standards for new sources of risk than for old ones-so that the costs to industry of the tough standards will be offset (perhaps more than offset) by barriers to 
competitive entry. ${ }^{37} \mathrm{~A}$ move like this (which happens to reflect regulatory reality) might satisfy political demands in all three periods: there would be an apparently strong response to availability-driven demand for instant overreaction to some incident; there would be a means to cater to the mobilization bias of industrial interests as salience subsides; there would be a reduction in the likelihood that a disastrous event will recur during one's political career.

The choice between strict legislative policy, on the one hand, and delegation of policy objectives to an administrative agency, on the other, turns on the duration of the availability effect as well as the probability of a damaging event recurring. The theory of availability owing to recency does not specify the rate at which overly intense feelings decay as the event recedes into the past, but this rate and the frequency of the sort of event in question determine two critical elements of the delegation/no delegation decision. The first is the proportion of the time that the issue will be salient and thus protected to some degree from mobilization bias. The second is the chance that today's elected officials will later be held accountable for some damaging event. If an event is unlikely to recur within the time horizon of a politician, and the politician (unlike constituents) is free of cognitive pathologies that lead to overestimation of the probability of recurrence, then the politically optimal policy is to do little or nothing of substance with respect to regulation but to cater to the overreaction and temporary mobilization of citizens who seek action. If the duration of the availability effect is short, lashing to the mast through delegation works for the reasons described above. But if the duration of the availability effect is sufficiently long that overreacting citizens remain mobilized throughout the protracted process of developing a policy through an administrative proceeding, there is likely to be excessive regulation. Thereafter, as the availability effect recedes, the politician is left with no political support from previously overreacting citizens (the issue is no longer salient for them) but with a residue of opposition from an industry that remains organized and has had to comply with unnecessary regulations.

One potentially safe escape for a politician facing these circumstances is to seek government subsidies for the risk-abatement policy. Subsidies will soften industry opposition and provide phantom benefits that overreacting citizens might see as larger than the costs. Perhaps the Superfund

37 The 1977 amendments to the Clean Air Act's new source performance standards are commonly interpreted as an application of this approach. See Bruce A. Ackerman \& William T. Hassler, Clean Air/Dirty Coal (1981); and McCubbins, Noll, \& Weingast, supra note 33. 
program for dealing with toxic waste dumps is an example of this strategy. Toxic chemicals are linked to cancer, and cancer and cancer policy seem to be perpetually salient issues. Environmental groups can rely on the representativeness heuristic to link toxic dumps to cancer and to extend the period of salience that follows some newsworthy event involving pollution from a dump. If relatively few dumps are really threats to public health, but large numbers of dumps are politically salient because of availability and representativeness, then the subsidization strategy keeps industry operating profitably, placates citizens, and avoids disaster. (Of course, the factual premises of this example are a matter of controversy, and there are other plausible explanations for a vigorous policy on toxic dumps. We are simply trying to illustrate one of the implications of cognitive theory for policy-making. There is, though, a major puzzle about toxic waste policy. If dumps are a major threat to public health, why has the government not been more active in passing clean-up costs on to industry - as it has been in the case of air and water pollutants and nuclear radiation? The cognitive theory suggests an answer.)

The last case to be considered is where the frequency of recurrence, assuming no regulatory intervention, is shorter than the time horizon of a politician. Here the politician has to trade off demands for excessive regulation against the self-interest of those responsible for creating the risk (they, presumably, want underregulation). If the duration of the availability effect is short, the politician's best available strategy is likely to be a compromise between these interests that is explicitly stated in legislation. Delegation to an agency would be attractive only if the agency could act rather quickly - before salience wanes and before another damaging event occurs. Thus, if delegation is observed, it should be accompanied by strict deadlines for decisions and a commitment to the agency of sufficient resources to make the deadline feasible.

If the period of salience is longer, delegation becomes more attractive, for it can result in an arrangement whereby the representatives of overreacting citizens, and those of self-interested risk producers, are forced to negotiate a compromise under the watchful eye of agencies and the courts. If the duration of the availability effect is long, participation in the policy development process in a regulatory agency will be likely to include both regulated firms and the most intense demanders for risk regulation. Thus, more balanced mobilization will encourage elected political officials to delegate the question of the overall stringency of a policy to an agency.

Our final observation about the optimal political strategy for dealing with risks is the general attractiveness of formulating policy choices in terms of risks of loss rather than as lotteries over gains. In part, loss 
aversion implies that citizens are easier to motivate when the issue is the threat of loss as opposed to the opportunity for gain. In addition, overreaction by citizens, if it can be orchestrated, is beneficial to political actors. If citizens can be made to attack a problem more intensively than its probability and the gravity of its damage warrant, the ultimate outcome-which is likely to be little or no damage-will cast favorable light on the responsible politicians. In other words, the very nonoccurrence of events will result in greater thanks to the politicians than they deserve. Moreover, by casting risk issues in negative, loss-aversive terms, a political leader is protected against effective challenge by someone adopting the same strategy. This argument is not symmetric: a negative formulation cannot as easily be attacked by a positive formulation because the shape of a citizen's valuation function depends on whether outcomes are gains or losses. That is, gains promise smaller changes in welfare than do losses; thus, they are less likely to be salient to the electorate, and less likely to lead to the mobilization of political activity.

\section{Concluding Remarks}

The cognitive theory of choice under uncertainty offers two sorts of insights of some relevance to analysts interested in the regulation of risk. First, people take shortcuts (use heuristics) that lead them to make mistakes about variables (such as probability) relevant to evaluating risk. Second, putting the mistakes aside, they approach risk in ways that depart from the norms and assumptions of conventional decision analysis.

The latter category of insights is, of course, much more interesting than the former because the observation that people make mistakes is hardly fresh or startling. Moreover, the cognitive theory seems to contain examples of all kinds of mistakes; for example, while availability may account for overreaction to a catastrophe, anchoring may explain underreaction. As yet, the theory cannot tell us very much about which mistakes are likely to occur in any given circumstance. Even at that, though, the cognitive psychologists' catalog of judgmental errors is a useful contribution, for several reasons. First, knowing something of the types, range, and causes and consequences of misjudgment can guide efforts toward more fruitful communication between the public and policymakers on matters of risk and its regulation, a matter currently (and righly) of much importance. Second, understanding that judgmental error is fairly regular can help avoid some policy pitfalls. Error is predictable as to kinds of mistakes and their direction, and it is likely to plague any decision maker, even a professional risk assessor. Recognizing these problems guards against such easy answers to problems of risk regulation as faithfully 
delegating the whole business to "experts." At the same time, an awareness of the problems enables experts to be sensitized to judgmental shortcomings in the course of their training, so as to make them more worthy of their name. ${ }^{38}$

Points like these are surely beyond contention, something that cannot be said of the implications of the other chief contribution of the cognitive theory-its claim that people think about risk, even when they do so free of miscalculation, in ways that depart from conventional decision theory. What the cognitive psychologists describe, after all (and as they well know), ${ }^{39}$ are citizens who are, from the conventional perspective, plainly irrational.

Here, the cognitive theory poses a real problem for conventional decision theorists and policymakers alike. For the first group, there seems to be "little hope for a theory of choice that is both normatively acceptable and descriptively adequate." ${ }^{40}$ For the second group, policymakers, the problem is that the measure of good policy becomes ambiguous: is it what the electorate persistently wants, or is it what conventional analysis persistently dictates? ${ }^{41}$

There is a way out of both of these binds, of course. Theorists and policymakers could simply accept the cognitive theory as the privileged viewpoint, such that the conventional ideas about rationality would become something of an anomaly, rather than (as at present) the other way around. Yet oddly enough, even the cognitive psychologists who have identified the binds in the first place appear to find such an alternative unattractive, perhaps even inappropriate..$^{42}$ In any event, so long as there is fundamental disagreement about whether we should follow the norms of conventional decision theory or instead accede to the norms at least implicit in prospect theory and other parts of the cognitive psychologists' descriptive picture, there will be fundamental disagreement about just what amounts to "good" regulation of risk.

${ }^{38}$ See James E. Krier, Risk and Design, in this issue.

${ }^{39}$ See, for example, Quattrone \& Tversky, supra note 5, at 734-35 (psychological account of political decision making describes behavior that diverges from standard definitions of rationality).

${ }^{40} \mathrm{Id}$. at 735 .

${ }^{41}$ See, for example, Slovic, Fischhoff, \& Lichtenstein, supra note 3, at 278.

${ }^{42}$ So Quattrone \& Tversky, supra note 5, at 735, say that the "descriptive failure of normative principles [drawn from conventional decision theory] . . . does not mean that people are unintelligent or irrational. The failure merely indicates that judgment and choice-like perception and memory - are prone to distortion and error." But why should the cognitive theorists label the behavior they study as revealing "distortion and error" simply because it departs from conventional norms? To say that is to say that the conventional norms are the "right ones," the privileged viewpoint. One could as well say that conventional theory simply has an otherwordly view of rationality. 
Policymakers, all politicians included, are likely to have a little less difficulty with this kind of disagreement than are those in more academic positions. True, political actors may at times "face the hard choice between going against their better judgment by using the public's assessment of risk (in which they do not believe) or going against the public's feeling by imposing policies that will be disliked." 43 On some occasions, though, they will surely be able to convince themselves that the most important thing, in the end, is that they act in a way that keeps them in their jobs. On even more occasions, they will find ways to satisfy the public and their own consciences all at the same time. As we suggested in Section III, means can be devised to give the public what it wants while, at the same time, answering to the rationality norms of conventional theory (or is it to the demands of another part of a policymaker's constituency?).

These last conclusions, it should be noticed, follow from our own application of conventional decision theory and its implications for the politics of regulation. Whatever the novel contributions of cognitive theory to the demand side of risk regulation, standard analysis readily suggests the sorts of policies that will be supplied in response. It is possible, of course, that our standard picture is simply wrong-possible, in particular, because it works from an assumption that, while citizens behave according to the cognitive psychologists' description of the world, politicians do not. ${ }^{44}$ What cuts in the other direction is that conventional theory does predict rather accurately the behavior of the regulatory system. Perhaps this is because it already incorporates, though sometimes in different jargon, a set of concepts similar to some of those devised by the cognitive theorists. "Availability," after all, is in many respects just another name for the "salience" of standard political theory, and to elaborate such heuristics as "representativeness" and "anchoring" might just be to codify what every successful politician (and every successful political analyst) knew long ago. But then again, maybe much was known long ago precisely because the research undertaken by the cognitive psychologists had seeped unnoticed into the common consciousness.

${ }^{43}$ Slovic, Fischhoff, \& Lichtenstein, supra note 3, at 278.

${ }^{44}$ See p. 749 supra. 